\title{
Zahnschäden durch Drogen: Gewinnerarbeit signalisiert Handlungsbedarf
}

Der mit insgesamt $12000 €$ dotierte Wrigley Prophylaxe Preis wurde zum 21. Mal im Rahmen der 2. Gemeinschaftstagung der DGZ und DGET mit der DGZPW und DGR2Z verliehen. Der 1. Platz geht an eine Arbeit aus München, die erstmals die verheerenden Auswirkungen der Szenedroge Crystal Meth auf die Zahngesundheit untersuchte. Den 2. Platz belegt eine Untersuchung aus Dresden zum erosionsprotektiven Potenzial von Pflanzenextrakten. Den Sonderpreis teilen sich ein Prophylaxeprojekt für Grundschulkinder in Wuppertal und eine Kooperationsinitiative zwischen Zahn- sowie Kinder- und Jugendärzten in Mönchengladbach.

\section{Crystal Meth ruiniert auch die Zahngesundheit \\ $\nabla$}

Dieses Jahr vergab die Jury den 1 . Platz an das Münchner Autorenteam um Dr. Dr. Niklas Rommel für eine Grundlagenstudie zu den Auswirkungen der Szenedroge Crystal Meth auf die Zahn-, Mund- und Kiefergesundheit. Die Gewinner sichern sich damit $6000 €$ Gewinnerprämie. Die Vergleichsstudie an 200 Patienten mit

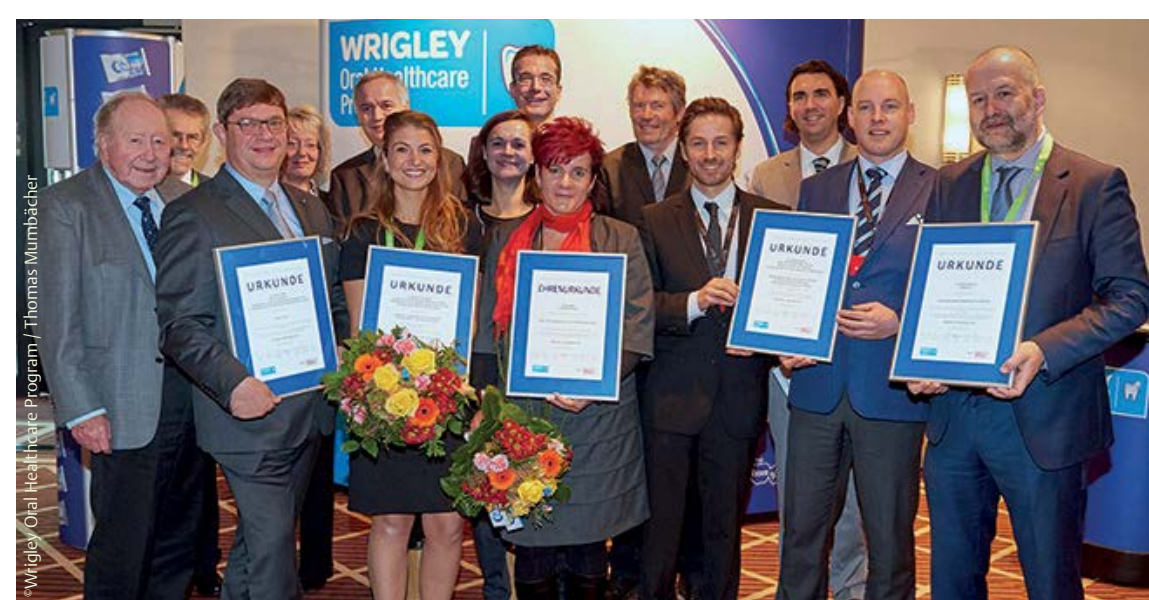

(von links) Prof. Klaus König (Jury, Nijmegen/Niederlande), Prof. Werner Geurtsen (Jury, Hannover), Dr. J. Zitzen (Sonderpreis, Mönchengladbach), E. Damann (Jury, Barmer GEK, Wuppertal), Prof. E. Schäfer (Jury, Münster), Dr. M.-T. Weber (2. Platz, Dresden), Laurence Étienne (Wrigley), Jens Christmann (Wrigley), A. Keller (Ehrung, Grünhain-Beierfeld), Prof. Joachim Klimek (Jury, Gießen), Dr. Dr. N. Rommel (1. Platz, München), Prof. Hendrik Meyer-Lückel (Jury, Aachen), Prof. Marco Kesting (1. Platz, München), Dr. A. Struve (Sonderpreis, Wuppertal). Nicht im Bild: Prof. Thomas Attin (Jury, Zürich).
Zähneknirschen. „Die prämierte Studie liefert erstmals eine solide Basis für die Entwicklung von Präventions- und Therapiekonzepten“, begründet Prof. Dr. Edgar Schäfer, Universität Münster, die diesjährige Preisvergabe.

\section{Erosionspräventives Potenzial von Pflanzenextrakten \\ $\nabla$}

Der 2. Platz ging mit $4000 €$ an die Arbeitsgruppe um Dr. Marie-Theres Weber, Dresden. Sie zeigte, dass Johannisbeerkrautund Oreganoextrakte vor erosiven Prozessen schützen können. Offenbar festigen pflanzliche Polyphenole die schützende Pellikel auf der Zahnoberfläche und reduzieren die Säurepermeabilität. Das Ergebnis der kontrollierten Studie an 12 Probanden nach Verwendung von Pflanzenextrakten oder Fluorid: Unter dem Einfluss der Pflanzenextrakte war die Pellikel dicker und schützte effektiver vor Erosionen - vergleichbar wie Fluoride oder besser. Die Studienergebnisse lassen vermuten, dass Extrakte aus Johannisbeerkraut und Oregano die Wirkung der Fluoride ergänzen. Beide Extrakte sind als Mundgesundheitspräparate in Apotheken und Drogerien erhältlich. Für eindeutige Aussagen bedarf es jedoch weiterer Forschung, so die Autoren.

\section{Sonderpreis für Prophylaxepro- jekt in der Grundschule \\ $\checkmark$}

Zudem verlieh die Jury zum 2 . Mal den mit $2000 €$ dotierten Sonderpreis „Niedergelassene Praxis und gesellschaftliches Engagement“. Diesen Preis teilen sich 2 engagierte Prophylaxeprojekte mit jeweils $1000 €$. Der Wuppertaler Zahnarzt Dr. Andreas Struve beeindruckte mit einem Grundschulprojekt, das er seit 2003 betreut. Einmal pro Jahr geben Struve und sein Praxisteam Prophylaxe-Unterricht bei Zweitklässlern, werten Ernährungstagebücher aus und führen bei allen Kindern einen Speicheltest zur Bestimmung des individuellen Kariesrisikos durch. Das Ergebnis erhalten die Eltern zusammen mit schriftlichen Therapieempfehlungen und einer Einladung zu einem Elternabend. Die Testergebnisse der letzten Jahre zeigen deutlich, dass viele Kinder ein sehr hohes Kariesrisiko haben. Frühzeiti- 
ge Prophylaxe und Aufklärung seien daher dringend notwendig, so Dr. Struve.

Ebenfalls mit dem Sonderpreis und einer Prämie von $1000 €$ ausgezeichnet wurden Dr. Jürgen Zitzen, Dr. Stephan Kranz, Dr. Dr. Ralf-Thomas Lange und Klaus Büssenschütt von der Zahnärzte Initiative Mönchengladbach ZIM. Seit 2008 kooperieren Zahnärzte mit Kinder- und Jugendärzten der Stadt in der Aktion ZIMkid. Was mit einer gemeinsamen Fortbildung begann, ist heute ein eingespieltes Netzwerk. Beispielsweise informieren Kinderärzte bei der zwischen dem 5 . und 7. Lebensmonat anstehenden U5 die Eltern zum Thema Zahngesundheit, weisen auf den 1. Zahn- arztbesuch hin und überreichen den Eltern den zahnärztlichen Kinderpass. Ein Erfolgsmodell par excellence, wie die Statistik zeigt: Seit Bestehen der Initiative nahm die Zahl der zahngesunden Kinder in Mönchengladbach von 60 auf über 70\% zu - mit steigender Tendenz. Jurymitglied Elke Damann, Barmer GEK, Wuppertal, war beeindruckt: „Zahn- und Kinderärzte arbeiten dort Hand in Hand. Kinderärzte schicken ihre kleinen Patienten früh zum Zahnarzt und sprechen bei den Untersuchungen das Thema Zahnpflege an. Das stärkt die Zahngesundheit von Anfang an.“

\section{Früh übt sich: Prophylaxe für die Kleinsten \\ $\checkmark$}

Mit einem Büchergutschein geehrt wurde das Spiel- und Lernprogramm „Abenteuer im Knabber-Schnapper-Lecker-Schmecker-Wunderland“ von Andrea Keller, Leiterin einer Kindertagesstätte im sächsischen Schwarzenberg. Die Sozialpädagogin entwickelte das Konzept, um Kinder im Alter von 2 bis 10 Jahren auf spielerische Weise für die tägliche Zahnpflege zu begeistern und ihnen ein Gefühl für den eigenen Körper zu vermitteln.

Aus einer Pressemitteilung des Wrigley Oral Healthcare Program 